\title{
Cerebellar Infarction Following Epidural Abscess after Epidural Neuroplasty
}

\author{
Hyun Yeong Lee ${ }^{1}$, Hui Sun Wang ${ }^{2}$, Seok Won $\mathrm{Kim}^{2}$, Chang II Ju${ }^{2}$ \\ Departments of ${ }^{1}$ Anesthesia and Pain Medicine, ${ }^{2}$ Neurosurgery, Chosun University College of Medicine, Gwangju, Korea
}

Epidural neuroplasty is found to be effective in removing fibrous tissue occurring in the epidural space for various reasons. We report a case of cerebellar infarction caused by epidural abscess after epidural neuroplasty. To the best of our knowledge, this is the first report of cerebellar infarction developed as a result of epidural abscess accompanying bacterial meningitis after epidural neuroplasty. We also discuss the etiology, pathogenesis, and prognosis of this rare pathologic entity.

Key Words: Neuroplasty $\cdot$ Epidural abscess $\cdot$ Infarction

\section{INTRODUCTION}

Remnant pain after surgical procedure for lumbar pathology may be due to postoperative epidural scarring. Percutaneous adhesiolysis might be a valuable technique in managing chronic, refractory low back and lower extremity pain, caused by epidural scarring ${ }^{5,7,10)}$. Following percutaneous adhesiolysis, several complications have been reported, such as dural puncture, infection, intravascular injection, hypotension and migration of catheter ${ }^{11,12}$. However, to the best of our knowledge, cerebellar infarction following epidural abscess immediately after epidural neuroplasty has not been reported yet. Here, we report a rare case of cerebellar infarction following epidural abscess after epidural neuroplasty, with a review of the relevant literature.

\section{CASE REPORT}

A 48-year old man with low back pain and pain in the left leg, secondary to unsuccessful surgery of spinal stenosis of L4L5, was admitted to our institute. A review of the patient's history showed that he had undergone decompressive laminotomy two years ago, and that he had suffered from diabetic

- Received: November 9, 2014 - Revised: March 3, 2015

- Accepted: March 4, 2015

Corresponding Author: Seok Won Kim, MD, PhD

Department of Neurosurgery, Chosun University College of Medicine,

365 Pilmun-daero, Dong-gu, Gwangju-city 501-717, Korea

Tel: +82-62-220-3126, Fax: +82-62-227-4575

E-mail: ns64902@hanmail.net/chosunns@chosun.ac.kr

$\otimes$ This is an Open Access article distributed under the terms of the Creative

Commons Attribution Non-Commercial License (http://creativecommons.org/ licenses/by-nc/3.0/) which permits unrestricted non-commercial use, distribution, and reproduction in any medium, provided the original work is properly cited. mellitus for 4 years, receiving treatment for this condition (Fig. 1). $\mathrm{He}$ had tried several therapeutic approaches, such as analgesic and anti-inflammatory drugs, physiotherapy, and epidural steroid injections, and all were unsuccessful. In our Anesthesia and pain medicine department, the patient underwent percutaneous epidural neuroplasty with hypertonic sodium chloride solution via Racz's catheter placed by the guidance of fluoroscopy, with verified location by contrast medium. Percutaneous adhesiolysis with bupivacaine and hypertonic saline was applied via Racz's catheter for 3 days. At the end of the third day, the patient was almost pain-free. Thus, Racz's catheter was removed and the patient was discharged. However, just three days after discharge, he was readmitted with fever, headache and confusion. He had focal neurological signs of stiff neck. His body temperature was $39.1^{\circ} \mathrm{C}$, WBC count rose to $22.500 /$ $\mu \mathrm{l}$, and erythrocyte sedimentation rate (ESR) increased to 100 $\mathrm{mm} / \mathrm{hr}$. With the presumptive diagnosis of pyogenic spondylitis, radiological studies were performed. Brain computed tomography (CT) scan showed non-specific findings (Fig. 2).
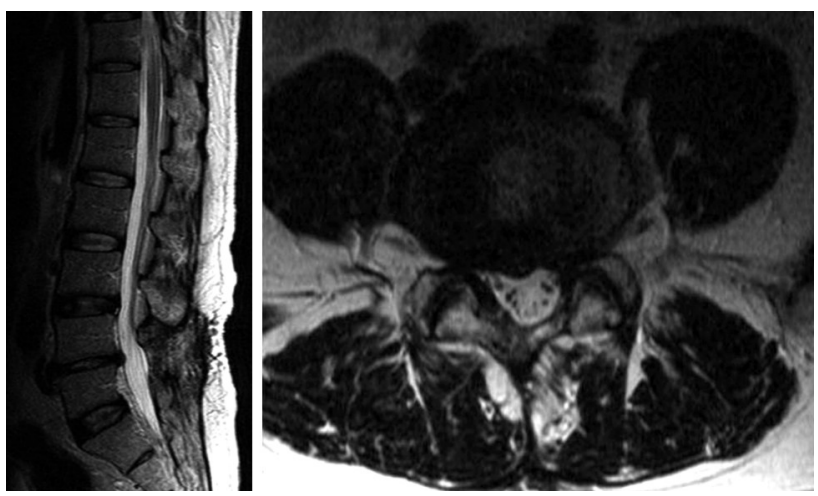

Fig. 1. T2 weighted sagittal and axial images show previous decompressive laminotomy state suggesting epidural scarring. 
Lumbar spine magnetic resonance imaging (MRI) revealed rim enhanced abnormal fluid collection suggesting purulent material at L5 level (Fig. 3). He was referred to Neurosurgical department for emergent surgery. Emergent abscess removal and wound irrigation were performed for bacterial culture. A yellowish purulent material was found and large amount of pus was released. Methicillin resistant staphylococcus aureus (MRSA) was identified in the pus. High dose of antibiotics, i.e. vancomycin and third generation cephalosporin, ceftriaxone (Hanmi Pharmaceuticals, Korea) were started, according to antibiotics susceptibility test. However, in spite of antibiotics therapy, the patient's level of consciousness had gradually deteriorated to deep drowsy state two days after the operation. To rule out bacterial meningitis, cerebrospinal fluid (CSF) analysis was performed. CSF cultures also showed the growth of MRSA. He was still febrile and developed recurrent seizures. Brain MRI revealed the presence of a left cerebellar infarction (Fig. 4). He was referred to Neurology department for anticoagulation medication. By 12 months after the admi-

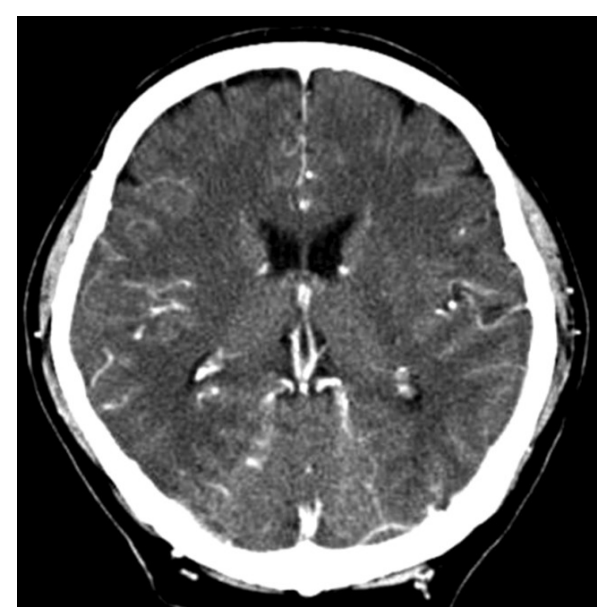

Fig. 2. Enhanced computed tomographic scan shows no significant abnormality.
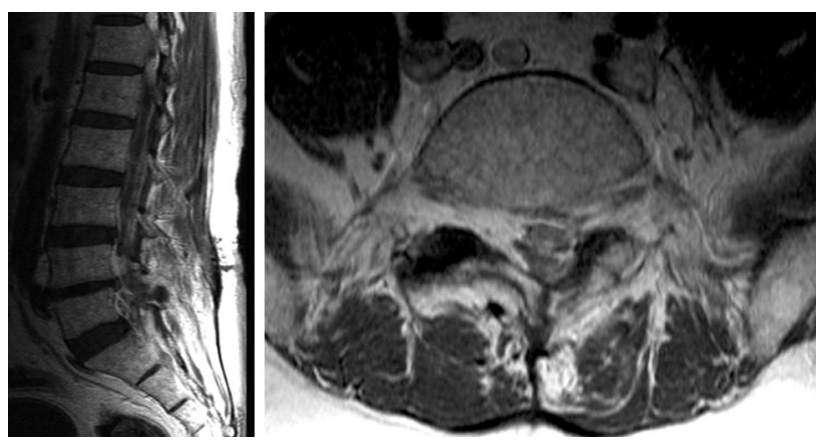

Fig. 3. Enhanced T1 weighted sagittal and axial images reveal rim enhanced abnormal fluid collection suggesting purulent material at L5 level. ssion, the patient could ambulate independently, without any difficulty, but complained of slight disturbance of coordination and equilibrium.

\section{DISCUSSION}

Racz's minimal invasive epidural catheter procedure, known as "epidural neuroplasty", is not utilized exclusively in patients suffering from failed back surgery syndrome, but is also increasingly applied to non-surgical back pain patients, to prevent chronification or deterioration, even in the neurosurgical field. Its hypothesized principle of action is local epidural lysis of adhesions, neurolysis of spinal nerve roots and local lavage of proinflammatory mediators, by repeated injection of local anesthetics, corticosteroids, hyaluronidase and hypertonic saline solution ${ }^{7,10,12)}$. However, adverse events are well known to occur in epidural neuroplasty. Thus, complications of epidural neuroplasty are due to the procedure itself or due to specific drug-related side effects. Unintended dural puncture, administration of the drugs to the subarachnoid or subdural space, catheter shearing, infection and severe hemodynamic instability during application are the most commonly observed adverse events ${ }^{11}$. Among them, epidural infection or abscess formation may be due to either hematogenous spread or extension of superficial skin infections. Immuncompromised patients, diabetic patients, steroid-dependant patients, and patients with malignancies are at higher risk for infection ${ }^{6}$. Epidural abscess formation may not be recognized at the beginning, since it is localized. In addition, fever, pain and increased white blood cell count may not always accompany this serious infection, although, in time, fever will rise as the abscess develops and meningismus signs may be observed. MRI evaluation is necessary to verify the extent of the problem, but it may not always reveal specific findings, especially in the beginning of abscess formation. In this patient, a high fever of $39^{\circ} \mathrm{C}$
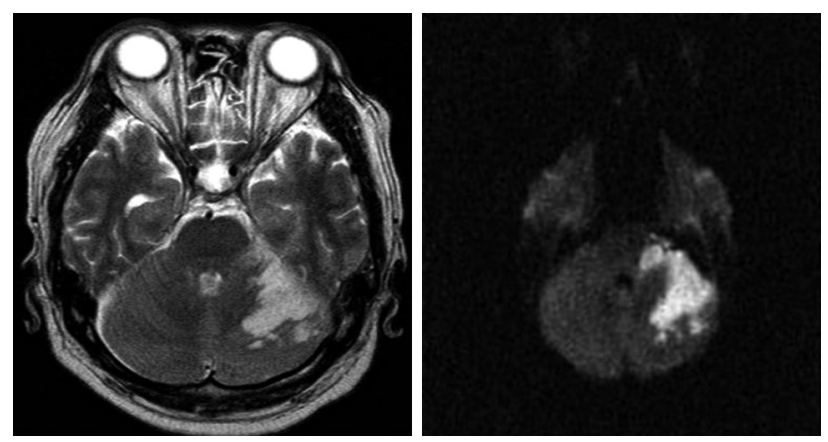

Fig. 4. T2 weighted axial and diffusion magnetic resonance images reveal acute left cerebellar infarction. 
developed on the sixth day of the procedure. The patient developed signs of meningitis with neck stiffness, headache, and confusion; though the brain CT did not reveal any abnormal findings of meningitis. Nagra et al. ${ }^{8)}$ reported the vast majority of CT scans being performed in patients with meningitis have normal findings. Cerebral vasculopathy is one of the major complications of bacterial meningitis. Large-size arteries at the base of the brain, medium-sized arteries, small pial and intraparenchymal arteries, as well as sinus and cortical veins might be affected. Alteration of the cerebral blood flow in the large-size vessels at the base of the brain has been previously shown, by means of ultrasound, in patient with bacterial meningitis ${ }^{1,2)}$. Brain infarction has been reported in bacterial meningitis caused by streptococcus pneumonia, Neisseria meningitides, Haemophilus influenza, Staphylococcus aureus, and other pathogens ${ }^{3,4)}$. However, the exact mechanism of vascular occlusion is still not clear. Probably most ischemic lesions can be explained by distal vasculitis and impaired cerebral blood flow. Occlusion of the great cerebral vessels has been reported to be caused by not only vasospasm but also by thrombosis. Usually, ischemic lesions affect the cerebral cortex, whereas cerebellum is rarely affected. The prognosis of bacterial meningitis with cerebrovascular complications is poor. In the study of Pfister et al. ${ }^{9)}$ only two of 13 patients recovered. Cerebral ischemia may lead to poor outcome, as a result of direct focal cerebral damage, as well as increased intracranial pressure due to cytotoxic edema.

\section{CONCLUSION}

Although epidural neuroplasty has been reported to be an effective treatment modality for low back pain from various causes, we should keep in mind that it can cause cerebellar infarction, following epidural abscess and bacterial meningitis.
The procedure must be performed by experienced hands in well-equipped clinics.

\section{REFERENCES}

1. Bentley P, Qadri F, Wild EJ, Hirsch NP, Howard RS: Vasculitic presentation of staphylococcal meningitis. Arch Neurol 64:17881789, 2007

2. Haring HP, Rötzer HK, Reindl H, Berek K, Kampfl A, Pfausler $\mathrm{B}$, et al: Time course of cerebral blood flow velocity in central nervous system infections. A transcranial Doppler sonography study. Arch Neurol 50:98-101, 1993

3. Igarashi M, Gilmartin RC, Gerald B, Wilburn F, Jabbour JT: Cerebral arteritis and bacterial meningitis. Arch Neurol 41:531535, 1984

4. Kastenbauer S, Pfister HW: Pneumococcal meningitis in adults: spectrum of complications and prognostic factors in a series of 87 cases. Brain 126:1015-1025, 2003

5. Lee F, Jamison DE, Hurley RW, Cohen SP: Epidural lysis of adhesions. Korean J Pain 27(1):3-15, 2014

6. Ma HJ, Kim IS: Clinical outcomes of spinal epidural abscess. Korean J Spine 9(1):6-11, 2012

7. Manchikanti L, Bakhit CE: Percutaneous lysis of epidural adhesions. Pain Physician 3:46-64, 2000

8. Nagra I, Wee B, Short J, Banerjee AK: The role of cranial CT in the investigation of meningitis. JRSM Short Rep 2(3):20, 2011

9. Pfister HW, Borasio GD, Dirnagl U, Bauer M, Einhäupl KM: Cerebrovascular complications of bacterial meningitis in adults. Neurology 42:1497-1504, 1992

10. Racz GB, Heavner JE, Trescot A: Percutaneous lysis of epidural adhesions-evidence for safety and efficacy. Pain Pract 8:277-286, 2008

11. Talu Gk, Erdine S: Complications of epidural neurolysis. A rerospective evaluation. Neuromodulation 6:237-247, 2003

12. Trescot AM, Chopra P, Abdi S, Datta S, Schultz DM: Systematic review of effectiveness and complications of adhesiolysis in the management of chronic spinal pain: an update. Pain Physician 10:129-146, 2007 